\title{
Listeria monocytogenes tyrosine phosphatases affect wall teichoic acid composition and phage resistance
}

\author{
Ran Nir-Paz ${ }^{1,2}$, Marcel R. Eugster ${ }^{3}$, Einat Zeiman², Martin J. Loessner ${ }^{3}$ \& Richard Calendar ${ }^{1}$ \\ ${ }^{1}$ Department of Molecular and Cell Biology, University of California, Berkeley, CA, USA; ${ }^{2}$ Department of Clinical Microbiology and Infectious \\ Diseases, Hadassah-Hebrew University Medical Center, Jerusalem, Israel; and ${ }^{3}$ Institute of Food, Nutrition and Health, ETH Zurich, Zurich, \\ Switzerland
}

\begin{abstract}
Correspondence: Ran Nir-Paz, Department of Clinical Microbiology and Infectious Diseases, Hadassah Hebrew University Medical Center, Jerusalem 91120, Israel. Tel.: +972 2 6776543; fax: +972 2 6419545; e-mail: ran.nirpaz@gmail.com
\end{abstract}

Received 18 July 2011; revised 19 October 2011; accepted 25 October 2011. Final version published online 18 November 2011.

DOI: $10.1111 / j .1574-6968.2011 .02445 . x$

Editor: Wolfgang Schumann

Keywords

phage resistance; tyrosine phosphatase; Listeria monocytogenes.

\begin{abstract}
Tyrosine phosphatase (PTP)-like proteins exist in many bacteria and are segregated into two major groups: low molecular weight and conventional. The latter group also has activity as phosphoinositide phosphatases. These two kinds of PTP are suggested to be involved in many aspects of bacterial physiology including stress response, DNA binding proteins, virulence, and capsule/cell wall production. By annotation, Listeria monocytogenes possesses two potential low molecular weight and two conventional PTPs. Using L. monocytogenes wild-type (WT) strain 10403S, we have created an in-frame deletion mutant lacking all four PTPs, as well as four additional complemented strains harboring each of the PTPs. No major physiological differences were observed between the WT and the mutant lacking all four PTPs. However, the deletion mutant strain was resistant to Listeria phages A511 and P35 and sensitive to other Listeria phages. This was attributed to reduced attachment to the cell wall. The mutant lacking all PTPs was found to lack $N$-acetylglucosamine in its wall teichoic acid. Phage sensitivity and attachment was rescued in a complemented strain harboring a low molecular weight PTP (LMRG1707).
\end{abstract}

choria monocytogenes.

\section{Introduction}

In recent years, accumulated data suggest that bacteria possess tyrosine kinases, phosphatases, and tyrosine phosphorylated proteins (Grangeasse et al., 2007). However, the role of such phosphorylation was elucidated only in a few species (Grangeasse et al., 2007). In Gram-negative bacteria, many tyrosine kinases and phosphatases were found (Bechet et al., 2009). In Escherichia coli, processes associated with cell wall modifications were suggested (Grangeasse et al., 2003; Peleg et al., 2005; Bechet et al., 2009). Phospho-proteome analysis of E. coli has revealed additional proteins phosphorylated on tyrosine, related to different cellular aspects including carbon metabolism and the glycolytic pathway (Macek et al., 2008). Additionally, other Gram-negative bacteria (such as Yersinia and Salmonella) were shown to have tyrosine phosphatases that are secreted into their host cells via a type III secretion system (YopH and SptP) (Murli et al., 2001; Cozzone, 2005; Yuan et al., 2005). These phosphatases are responsible for the manipulation of the host response to the benefit of the pathogen. In Gram-positive bacteria, tyrosine phosphorylation machinery was documented in both pathogenic bacteria (e.g. Streptococcus pneumoniae and Staphylococcus aureus) (Grangeasse et al., 2007; Bechet et al., 2009) and nonpathogenic bacteria (e.g. Bacillus subtilis and Lactococcus lactis) (Grangeasse et al., 2007; Bechet et al., 2009).

Interestingly, in Mycobacterium tuberculosis, it was thought that the two secreted protein tyrosine phosphatases (PTP), PtpA and PtpB, do not have a bacterial kinase counterpart and are thus responsible mostly for host manipulation (Chao et al., 2010). However, a recent finding suggests that PtpA is phosphorylated on tyrosine by a newly identified nonconservative tyrosine kinase, PtkA (Bach et al., 2009; Chao et al., 2010).

Listeria monocytogenes is a ubiquitous facultative intracellular Gram-positive bacterium that causes invasive devastating disease mainly in older people, pregnant women (leading to abortion and fetus loss), newborns, and 
immunocompromised hosts (Siegman-Igra et al., 2002; Guevara et al., 2009). Interestingly, L. monocytogenes has four PTPs without known adjacent kinase genes. These phosphatases belong to two major types - two low molecular weight PTPs and two conventional PTPs (Kastner et al., 2011). Recently, it was suggested that the two conventional PTPs belong to a group of enzymes that includes the M. tuberculosis PtpB (Beresford et al., 2010; Kastner et al., 2011). This group of phosphatases is active on phosphoinositides as well as on tyrosine phosphates (Koul et al., 2000; Beresford et al., 2010). Lower phosphorylated serine/threonine activity was noted as well (Beresford et al., 2010). In Listeria, it was shown that a mutant of LO28 strain deficient in one PTP (lipA) had lower virulence and lower bacterial counts in target organs (Kastner et al., 2011). Additionally, it was suggested that such PTPs might serve as a target for new antibiotics, mainly for the intracellular pathogen M. tuberculosis (Grundner et al., 2007; Beresford et al., 2009; Zhou et al., 2010). Thus, understanding the role of PTPs in L. monocytogenes should also elucidate its role in other pathogenic and intracellular bacteria.

\section{Materials and methods}

\section{Bacterial strains}

The L. monocytogenes strains used (see Table 1) were a wild-type strain (WT), 10403S, or a strain containing an in-frame deletion of each of the PTP (DP-L5359). These deletions were generated by sequential deletion of each of the phosphatases using splice-overlap extension (SOE)PCR and allelic exchange, as described elsewhere (Camilli et al., 1993) using the primers in the Supporting Information, Table S1. Complemented strains harboring only one of each of the phosphatases were generated using the pPL2 integrational vector (Lauer et al., 2002) and the primers in Table S1 to synthesize the PTP genes. Listeria monocytogenes DP-L861, also known as Mack (Hodgson, 2000), was used for phage propagation.

\section{Bioinformatics}

Nucleotide and amino acid sequence analyses and interpretation were carried out using Vector NTI Advance (Invitrogen, Basel, Switzerland). Pairwise sequence align- ments were made using the BLASTn, BLASTP, and tBLAST programs available at the NBCI website. The multiple alignment was made using CLustalW2 (http://www.ebi. ac.uk/Tools/msa/clustalw2/). The program BOXSHADE 3.21 (http://www.ch.embnet.org/software/BOX_form.html) was used to generate white letters on black boxes for residues that are identical and white letters on gray boxes for residues that are similar in sequences.

\section{Bacterial intracellular growth curves}

Bacterial intracellular growth curves were determined as described previously (Portnoy et al., 1988). Briefly, $2 \times 10^{6}$ bone marrow-derived macrophages (BMDM) were infected with $4 \times 10^{5} \mathrm{CFU}$ of L. monocytogenes from an overnight culture. Thirty minutes after the addition of bacteria, macrophage monolayers were washed with PBS. One hour postinfection, gentamicin was added to $50 \mu \mathrm{g} \mathrm{mL}^{-1}$ to kill the extracellular bacteria. At different time points postinfection, three coverslips were taken and washed with water to lyse host cells. Bacteria recovered from each coverslip were plated on brain heart infusion (BHI) plates, and the number of CFU was determined.

\section{Phage growth}

A511 was prepared according to Loessner \& Scherer (1995). A118 and U153 were prepared as described for A118 by Loessner et al. (2000), except that the host strain was DP-L861. P35 (Hodgson, 2000) was prepared as a plate stock, using Luria-Bertani (LB) plates supplemented with $5 \mathrm{mM} \mathrm{CaCl}_{2}$. The stock was sterilized by filtration through pores of $0.4 \mu \mathrm{m}$ diameter.

\section{Phage adsorption assays}

Standing cultures of bacteria were grown in BHI overnight at $30{ }^{\circ} \mathrm{C}$. The cell concentrations were $>10^{8} \mathrm{~mL}^{-1}$; $40 \mu \mathrm{L}$ of cells was mixed with $1 \mu \mathrm{L}$ of A511 $\left(4 \times 10^{7} \mathrm{~mL}^{-1}\right)$ and $1 \mu \mathrm{L}$ of $0.5 \mathrm{M} \mathrm{CaCl}_{2}$. The mixture was incubated for $15 \mathrm{~min}$ at $30{ }^{\circ} \mathrm{C}$, and the bacteria were removed by centrifugation. We assayed phage remaining in the supernatant on BHI plates, using DP-L861 as indicator.

Phage plaquing efficiency was determined by titrating 100-fold dilutions of various Listeria phages (A511, P35,

Table 1. Listeria strains used in this study

\begin{tabular}{|c|c|c|c|c|c|c|}
\hline Strain & 104035 & DP-L5359 & DP-L5412 & DP-L5413 & DP-L5414 & DP-L5415 \\
\hline Description & WT strain & $\begin{array}{l}\text { 10403S, } \Delta \text { LMRG2037, } \\
\Delta \text { LMRG0947, } \\
\Delta \text { LMRG1082, } \\
\Delta \text { LMRG1707 }\end{array}$ & $\begin{array}{l}\text { DP-L5359 } \\
\text { tRNA }^{\text {arg.::LMRG0947 }}\end{array}$ & $\begin{array}{l}\text { DP-L5359 } \\
\text { tRNA }^{\text {arg.:.LMRG } 1082}\end{array}$ & $\begin{array}{l}\text { DP-L5359 } \\
\text { tRNA }^{\text {arg::LMRG2037 }}\end{array}$ & 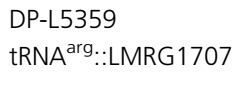 \\
\hline
\end{tabular}


U153, and A118) with the strains described in this study. The numbers of plaques were compared with the numbers obtained with the WT strains 10403S and DP-L861. Plaques were enumerated after incubation at $30{ }^{\circ} \mathrm{C}$ for 24 and $72 \mathrm{~h}$.

Sensitivity of L. monocytogenes to bacteriophage lysin was determined as was previously described (Loessner et al., 1996). Briefly, stationary L. monocytogenes strains were washed twice with $\mathrm{PBS}$ and resuspended in $50 \mathrm{mM}$ $\mathrm{Na}_{2} \mathrm{HPO}_{3}$ at $\mathrm{A}_{600 \mathrm{~nm}}$ of 1 . Then, strains were exposed to A511 Ply (bacteriophage lysin) (Loessner et al., 1996) at a final concentration of $1 \mathrm{U} \mathrm{mL}^{-1}$ and were followed for change at optical density (OD) $\mathrm{A}_{600 \mathrm{~nm}}$ absorbance for $90 \mathrm{~min}$.

\section{Preparation of cell walls}

Cell walls were purified as previously described (Fiedler et al., 1984; Valyasevi et al., 1990; Eugster \& Loessner, 2011). Bacterial strains were grown in BHI broth to an $\mathrm{A}_{600 \mathrm{~nm}}$ of 0.8 and inactivated by heating to $100{ }^{\circ} \mathrm{C}$ for $20 \mathrm{~min}$. Cells were harvested by centrifugation $(7000 \mathrm{~g}$, $\left.10 \mathrm{~min}, 4^{\circ} \mathrm{C}\right)$, resuspended in SM buffer $(100 \mathrm{mM} \mathrm{NaCl}$, $10 \mathrm{mM} \mathrm{MgSO}_{4}, 10 \mathrm{mM}$ Tris- $\mathrm{HCl}, \mathrm{pH}$ 7.5), and disrupted by passing through a French Press at $270 \mathrm{MPa}$. Unbroken cells were sedimented by centrifugation at $1400 \mathrm{~g}$ for $5 \mathrm{~min}$, and crude cell walls were washed twice with water and resuspended in SM buffer. The crude cell wall fraction was treated enzymatically with DNase and RNase at room temperature for $3 \mathrm{~h}$ and subsequently incubated with proteinase $\mathrm{K}$ for another $2 \mathrm{~h}$ (enzyme concentrations: $100 \mu \mathrm{g}$ $\mathrm{g}^{-1}$ wet crude cell walls). Further, cell walls were boiled in $4 \%$ sodium dodecyl sulfate (SDS) for $30 \mathrm{~min}$ and recovered by centrifugation $\left(30000 \mathrm{~g}, 30 \mathrm{~min}, 20^{\circ} \mathrm{C}\right.$ ), and the pellet was washed five times with water to remove residual SDS. The resulting preparation was lyophilized and used for the determination of total cell wall phosphate content.

\section{Determination of cell wall phosphate content}

To measure total cell wall phosphate content, samples were assayed as published earlier (Eugster \& Loessner, 2011). A $10-\mu \mathrm{L}$ sample of a $10 \mathrm{mg} \mathrm{mL}^{-1}$ purified cell wall suspension was first digested oxidatively using a NANOCOLOR ${ }^{\circledR}$ NanOx Metal (Macherey-Nagel) according to the manufacturer's protocol. Then, total phosphorus was determined photometrically by the use of a phosphate test kit (Spectroquant ${ }^{\circledR}$ Phosphate Test; Merck) as described by the manufacturer. To assure the accuracy and reliability of the results, a calibration curve was obtained with aqueous dilutions of a $1000 \mathrm{mg} \mathrm{L}^{-1}$ phosphate standard solution (VWR). All samples were decomposed and measured in triplicate.

\section{Detection of $\boldsymbol{N}$-acetylglucosamine in wall teichoic acids}

Wheat germ agglutinin (WGA)-Alexa Fluor $594^{\circledR}$ conjugate (Invitrogen) was used for the detection of $\mathrm{N}$-acetylglucosamine (GlcNAc) in wall teichoic acids (WTA) of Listeria cells. This lectin recognizes terminal GlcNAc substituents in cell wall polymers, such as WTA on the surface of L. monocytogenes (Wright, 1984; Loessner et al., 2002; Eugster \& Loessner, 2011). Binding assays with labeled WGA were performed as described elsewhere (Loessner et al., 2002; Eugster \& Loessner, 2011). Bacterial cells were harvested in late log phase by centrifugation and resuspended in 1/10th volume of PBST buffer (120 mM NaCl, $50 \mathrm{mM}$ phosphate, and $0.1 \%$ Tween 20, $\mathrm{pH} 8.0) ; 100 \mu \mathrm{L}$ cells and $50 \mu \mathrm{L}$ of Alexa Fluor $594^{\circledR}$ WGA solution $\left(0.1 \mathrm{mg} \mathrm{mL}^{-1}\right)$ were mixed and incubated for $10 \mathrm{~min}$ at $25^{\circ} \mathrm{C}$. Cells were removed from labeling solution by centrifugation (12 $000 \mathrm{~g}, 1 \mathrm{~min}$ ) and washed twice in PBST buffer. After washing, the cells were examined by fluorescence microscopy (Leica TCS SPE; Leica, Heerbrugg, Switzerland). Additionally, the presence of GlcNAc was tested using GFP-labeled cell wall-binding domain (CBD) of bacteriophage endolysin PlyP35 (HGFP-CBDP35), which specifically recognizes GlcNAc residues in Listeria WTA (Eugster et al., 2011). Binding assays with HGFP-CBDP35 were performed as described earlier (Loessner et al., 2002; Schmelcher et al., 2010; Eugster et al., 2011).

\section{Statistical analysis}

All experiments were repeated at least twice to confirm reproducibility. Categorical data were compared using the chi-square test or the Fisher's exact test when appropriate. Continuous variables were compared using the MannWhitney $U$-test or Student's $t$-test if number of repetitions was $<5$.

\section{Results}

\section{Bioinformatics analysis of tyrosine phosphatases in L. monocytogenes}

Listeria monocytogenes harbors four putative tyrosine phosphatases (LPTP): two of them belong to the low molecular weight phospho-tyrosine phosphatases and are annotated as LMRG2037 (LptpA1) and LMRG1707 (LptpA2) (Fig. 1). An additional two belong to the conventional weight phospho-tyrosine phosphatases and are annotated as LMRG0947 (LptpB1; lipA, LMO1800 in L. monocytogenes strain EGDe) and LMRG1082 (LptpB2, 
LMO1935 in L. monocytogenes strain EGDe) and described in detail recently (Beresford et al., 2010; Kastner et al., 2011).

All four tyrosine phosphatases are highly conserved within all strains of Listeria species that were fully sequenced to date (Table 2). All four PTP-coding genes were found in all sequenced strains of Listeria except for LptpA2, which was missing in the published fully sequenced L. monocytogenes LO28 isolate (serotype 1/2c). In the only sequenced Listeria grayi isolate, both conventional PTPs are missing; however, the genome of this isolate contains two other conventional PTPs that have no homologs in other Listeria strains. An operon that is homologous to the operon of LptpA2 was found in $B$. subtilis (Musumeci et al., 2005) and in other Gram-positive bacteria such as S. aureus (Musumeci et al., 2005). Additionally, LptpA1 has $51 \%$ amino acid similarity and $31 \%$ aa identity to PtpA of M. tuberculosis (Fig. 1a) and is suggested to be a secreted PTP (Bach et al., 2008).

\section{Creation of tyrosine phosphatases deletion mutants}

To study the specific role of each phosphatase and to prevent a possible cross-reactivity and specificity as is suggested by the sequence homology, we have created a L. monocytogenes mutant lacking all PTPs (DP-L5359). This was achieved by sequential deletions of all four phosphatases in the WT strain 10403S. We also have created single gene complemented strains, using the pPL2 integration vector as previously described (Lauer et al.,
2002). All strains used in this study are presented in Table 1.

\section{Tyrosine phosphatases in a variety of conditions in L. monocytogenes}

We looked for differences in L. monocytogenes physiology between the WT and the PTPs knock-out strain. We did not observe a growth defect in BHI or LB at either 37 or $30{ }^{\circ} \mathrm{C}$ (data not shown except for BHI $30{ }^{\circ} \mathrm{C}$, Fig. 2a). In a previous report, it was suggested that $B$. subtilis lacking a low molecular PTP is more sensitive to ethanol stress (Musumeci et al., 2005). However, the DP-L5359 grew without significant difference compared with WT in the presence of 5\% ethanol (Fig. 2b). Additionally, DP-L5359 was able to resist oxidative stress $\left(100 \mathrm{mM} \mathrm{H}_{2} \mathrm{O}_{2}\right)$ more efficiently than the WT (Fig. 2c). To assess whether cell wall integrity is impaired, we looked at differences in susceptibility to mutanolysin of the different L. monocytogenes strains. DP-L5359 was more resistant to mutanolysin, as was noticed by reduced clearance of turbidity after exposure to $100 \mathrm{mM}$ mutanolysin (Fig. 2d). No differences were observed after exposure of strains to lysozyme (Fig. S1). DP-L5359 also had a small swarming motility defect, as was shown by its reduced ability to spread on BHI soft agar $(10 \%$ reduction in motility, $P=0.045)$. Growth curves of both WT and PTPs knock-out strain (DP-L5359) in BMDM revealed similar growth curves without any major growth defect for the mutant (Fig. 2e). No differences in growth curves were observed in IFN- $\gamma$ activated BMDM (Fig. S2). Similarly, no difference in
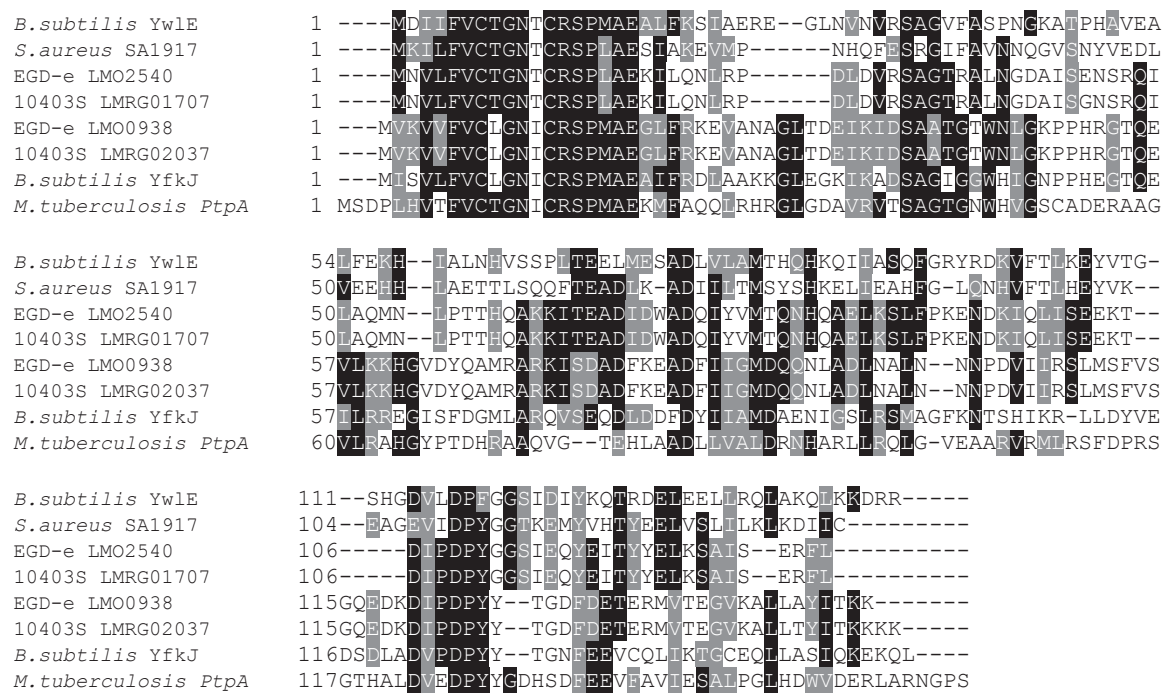

Fig. 1. The amino acid alignments of two Listeria monocytogenes WT (10403S and EGD-e) PTP. Comparison of the two low molecular weight PTP of both L. monocytogenes with those of Bacillus subtilis YwlE (gil2636218) and YfkJ (gil2633112); Mycobacterium tuberculosis PtpA (gil1261936l); and Staphylococcus aureus low molecular weight PTP (gil14247888'). 
Table 2. Genomes of Listeria species harboring putative tyrosine phosphatases by annotation

\begin{tabular}{|c|c|c|c|c|c|}
\hline & $\begin{array}{l}\text { Names of sequenced } \\
\text { genomes in the } \\
\text { NCBI database }\end{array}$ & $\begin{array}{l}\text { LptpA1 LMRG } 2037 \\
\text { (LMO 0938) }\end{array}$ & $\begin{array}{l}\text { LptpA2 LMRG } 1707 \\
\text { (LMO 2540) }\end{array}$ & $\begin{array}{l}\text { LptpB1 LMRG } 0947 \\
\text { (LMO 1800) }\end{array}$ & $\begin{array}{l}\text { LptpB2 LMRG } 1082 \\
\text { (LMO 1935) }\end{array}$ \\
\hline $\begin{array}{l}\text { L. monocytogenes serotype } \\
1 / 2 \text { a (9 genomes) }\end{array}$ & $\begin{array}{l}08-5578 \\
08-5923 \\
104035 \\
\text { EGD-e } \\
\text { F6900 } \\
\text { N3-165 } \\
\text { J0161 } \\
\text { J2818 } \\
\text { F6854 }\end{array}$ & 9 & 9 & 9 & 9 \\
\hline $\begin{array}{l}\text { L. monocytogenes serotype } \\
1 / 2 \text { b (4 genomes) }\end{array}$ & $\begin{array}{l}J 1-194 \\
J 1-175 \\
J 2-064 \\
R 2-503\end{array}$ & 4 & 4 & 4 & 4 \\
\hline $\begin{array}{l}\text { L. monocytogenes serotype } \\
1 / 2 c \text { ( } 2 \text { genomes) }\end{array}$ & $\begin{array}{l}\mathrm{R} 2-561 \\
\mathrm{LO} 28\end{array}$ & 2 & 1 & 2 & 2 \\
\hline $\begin{array}{l}\text { L. monocytogenes serotype } \\
\text { 3a (1 genome) }\end{array}$ & Finland 1988 & 1 & 1 & 1 & 1 \\
\hline $\begin{array}{l}\text { L. monocytogenes serotype } \\
4 a \text { ( } 2 \text { genome) }\end{array}$ & $\begin{array}{l}\mathrm{HCC} 23 \\
\mathrm{M} 7\end{array}$ & 2 & 2 & 2 & 2 \\
\hline $\begin{array}{l}\text { L. monocytogenes serotype } \\
4 b \text { ( } 5 \text { genomes) }\end{array}$ & $\begin{array}{l}\text { F2365 } \\
\text { H7858 } \\
\text { HPB2262 } \\
\text { J1816 } \\
\text { N1-017 } \\
\text { scottA } \\
\text { Clip80459 }\end{array}$ & 7 & 7 & 7 & 7 \\
\hline $\begin{array}{l}\text { L. monocytogenes serotype } \\
\text { 4c (1 genome) }\end{array}$ & J2-071 L99 & 2 & 2 & 2 & 2 \\
\hline Listeria innocua (1 genome) & Clip11262 & 1 & 1 & 1 & 1 \\
\hline Listeria seeligeri (1 genome) & SLCC3954 & 1 & 1 & 1 & 1 \\
\hline Listeria welshimeri (1 genome) & SLCC5334 & 1 & 1 & 1 & 1 \\
\hline Listeria grayi (1 genome) & DSM 20601 & 1 & 1 & 0 & 0 \\
\hline
\end{tabular}

Distribution of putative tyrosine phosphatases according to the annotation of 31 sequenced Listeria species available at the NCBI database. Similarity was identified using BLASTp suite (http://blast.ncbi.nlm.nih.gov/Blast.cgi). For each putative tyrosine phosphatase, the L. monocytogenes 104035 genome numbering was used (EGDe LMO number was provided in parenthesis) and a number of genomes that harbor the putative protein are noted. The full list of strains, serotypes and GenBank access numbers can be found in Table S2.

growth curve was also observed in epithelial cell lines (CaCo2 and HepG2, data not shown). Additionally, DPL5359 had no virulence defect compared with the WT 10403S in the mouse model of infection (Fig. S3).

\section{Phage susceptibility}

Bacteriophages have a life cycle that involves many bacterial physiological aspects: phages adsorb to the bacterial cell wall, then penetrate into the cell, replicate using bacterial machinery for both nucleic acids and proteins, mature and reassemble new phages, break the cell wall using lysozyme-like enzymes, and release progeny virions. Therefore, phages are useful tools for evaluating possible changes affected by many processes. We tested our WT
(10403S strain), deletion mutant, and complemented strains for susceptibility to Listeria phages. No differences were found using phages U153 and A118. However, A511 showed an extremely reduced plaquing efficiency on the PTPs deletion mutant DP-L5359, with phenotype restoration in the strain complemented with LMRG1707 LptpA2 (Fig. 3a). A similar observation was noted with phage P35 (data not shown). Thus, the lack of PTPs blocks the phage infection cycle, and LptpA2 restores phage growth. Both WT and knock-out strains lyse at the same rate with exposure to the purified A511 lysin (Fig. 3b), suggesting that release of the phages is not affected. To see specifically whether phage attachment is crucial for these differences, we have used a phage adsorption assay. Exposing phages to $10403 \mathrm{~S}$ resulted in almost complete elimination of 
(a)

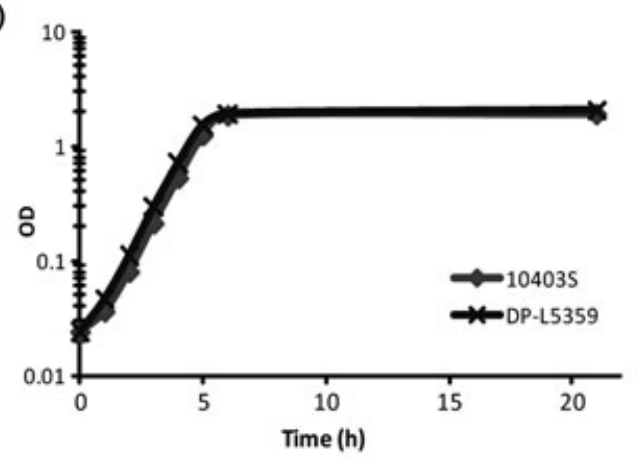

(c)

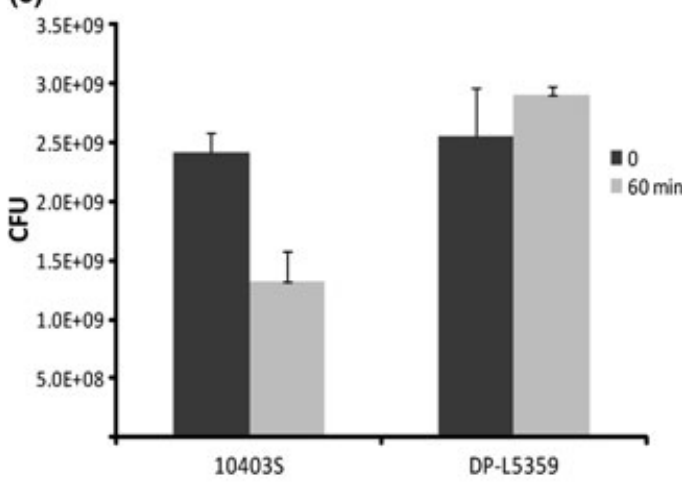

(b)

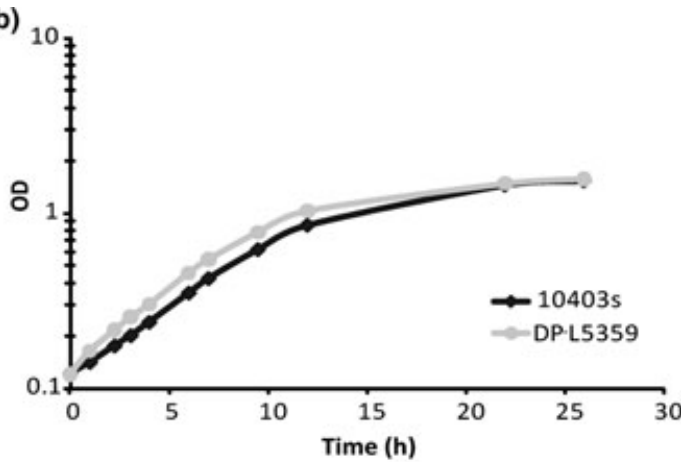

(d)

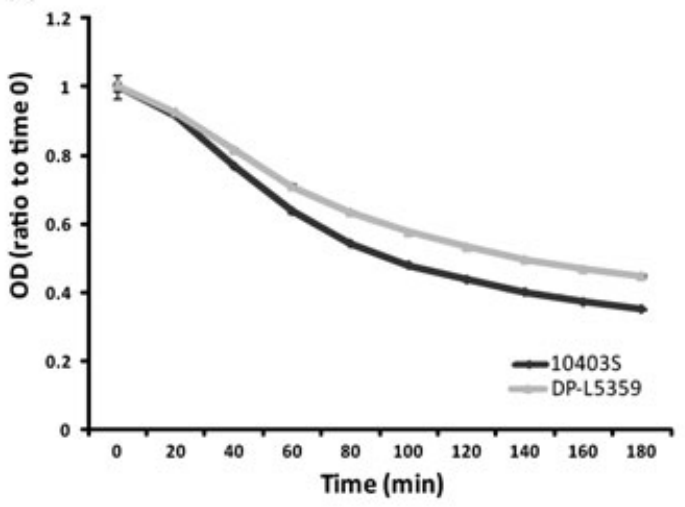

(e)

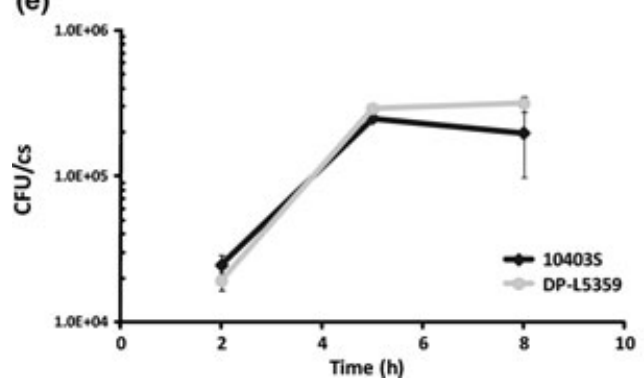

Fig. 2. Effect of tyrosine phosphorylation on Listeria physiology - (a) L. monocytogenes $10403 \mathrm{~S}$ and DP-L5359 were grown in BHI broth $30{ }^{\circ} \mathrm{C}$, in orbital shaker, until reaching stationary phase. The experiment was carried out in triplicate, and error bars represent SD of three experiments. (b) L. monocytogenes 104035 and DP-L5359 were grown in BHI broth $30{ }^{\circ} \mathrm{C}$ supplemented with $5 \%$ ethanol. DP-L5359 grew without significant difference from WT in the presence of $5 \%$ ethanol. (c) Approximately $2 \times 10^{9}$ CFU of both WT (10403S and PTPs KO mutant DP-L5359) was exposed to $100 \mathrm{mM} \mathrm{H}_{2} \mathrm{O}_{2}$ for $60 \mathrm{~min}$. After $60 \mathrm{~min}$, bacteria in solution were enumerated. Experiments were repeated three times, and error bars represent the SD of these triplicates. Almost 45\% reduction in the viability of 104035 was noticed compared with no in DP-L5359

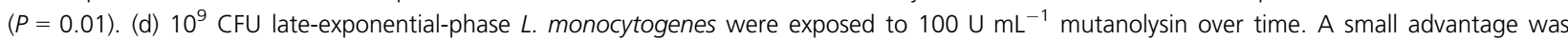
noticed for the PTPs deletion mutant (DP-L5359) over the WT (10403S) in all time points after exposure to mutanolysin $(P<0.01)$. Neither strain showed any change in absorbance up to 220 min when exposed to buffer alone ( $50 \mathrm{mM} \mathrm{NaHPO} 3 \mathrm{pH} 6.8$ ). A600 nm OD was measured at each time point, and SD represents a SD of three experiments. (e) Intracellular growth curves of WT L. monocytogenes (black) and the DP-L5359 deletion mutant (gray) in BMDM. Error bars represent one standard deviation.

phage from solution, while only very low numbers of phage were eliminated by exposing phage to DP-L5359 (Fig. 3c). This suggested to us that some differences in cell wall might be responsible for this phenotype. Interestingly, attachment was almost completely restored by one complemented strain (DP-L5415; complementation of the
LMRG1707 LptpA2) and less so ( 25\%) by another complemented strain (DP-L4212; complementing with the LMRG0947 LptpB1/lipA). No complementation of attachment was observed in the other complemented strains. Thus, LptpA2 is responsible for the restoration of cell wall attachment by A511. 


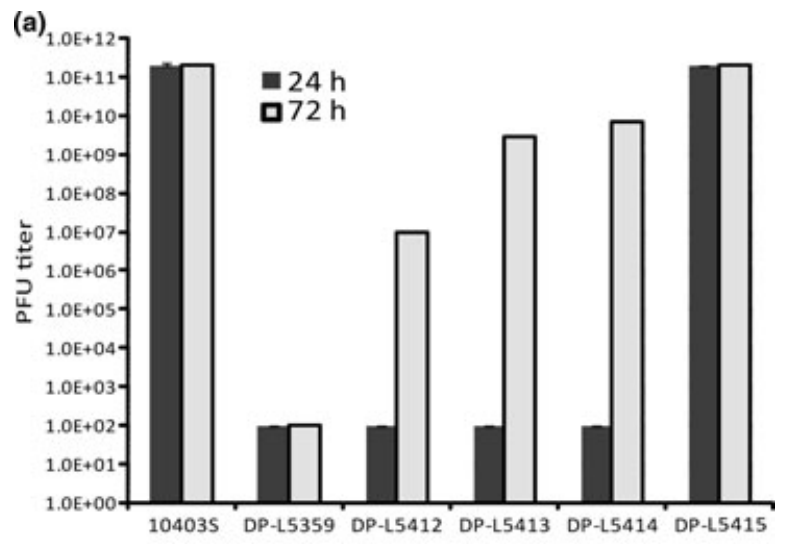

(b)

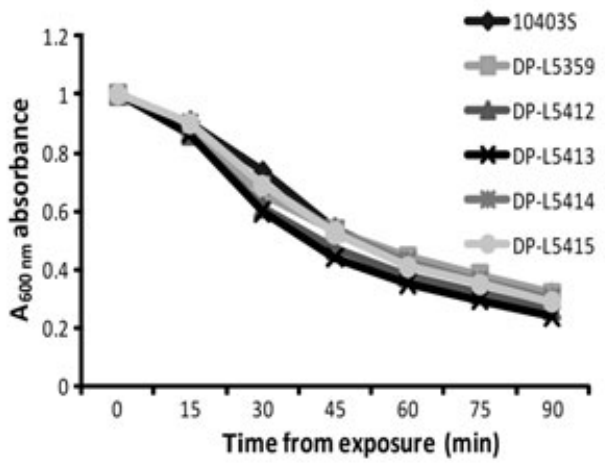

(c)

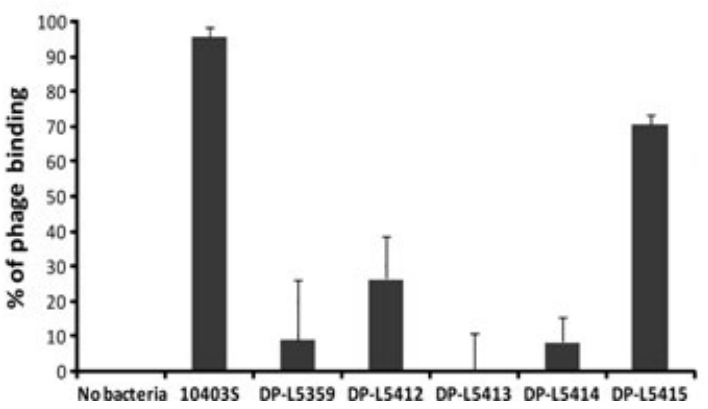

Fig. 3. The susceptibility of Listeria monocytogenes 10403S, PTPS deletion mutant DP-L5359, and complemented strains to Listeria phage A511. (a) Titer of PFU observed $24 \mathrm{~h}$ (dark columns) and $72 \mathrm{~h}$ (light gray columns) after exposing different $L$. monocytogenes strains to the same number of A511 (1.2 × $\left.10^{11} \mathrm{PFU} \mathrm{mL}{ }^{-1}\right)$. After $24 \mathrm{~h}$ only, complementation with LptpA2 (DP-L5415) enabled to restore WT phenotype. After $72 \mathrm{~h}$ of exposure to phage A511 of different L. monocytogenes, complementation with LptpB2 and LptpA1 partially restored the susceptibility. (b) Lysis of L. monocytogenes by purified A511 lysin is not influenced by the PTPs. Stationary L. monocytogenes strains were washed twice with PBS and resuspended in $50 \mathrm{mM} \mathrm{Na}_{2} \mathrm{HPO}_{3}$ at $A_{600 \mathrm{~nm}}$ of 1 . Then, strains were exposed to A511 ply (Loessner et al., 1996) for different time periods. Similar lysis was observed in WT, DP-L5359, and four complemented strains $(P>0.1$ comparing different strains to WT). (c) Phage adsorption can be only partially restored by only 2 PTPs. LptpA2 (DP-L5415) restores $\sim 70 \%$ of WT absorption $(P=0.001)$ and LptpB1 (DP-L5412) can minimally restore absorption $(20 \% P=0.14)$. The two other complemented strains did not restore adsorption at all compared with DP-L5359 $(P=1)$. Error bars represent standard deviation of three repetitions. The percent of phage absorbed was correlated with the number of phages in the solution when phages were incubated without bacteria for the same time.

\section{Identifying cell wall changes}

Taken together, the phage experiments and the changes after exposure of L. monocytogenes to mutanolysin suggested that changes in cell wall glycopeptide might be involved. First, we have looked for changes in the teichoic acid contents of the cell wall. Purified cell walls of 104035 and deletion mutant DP-L5359 were analyzed for total phosphorus to show the presence of teichoic acids in the cell walls. Both strains provided similar values indicating similar WTA content (Fig. S4). Thereafter, we looked for changes in cell wall glycosylation. Binding of fluorescently labeled WGA, a lectin that specifically binds terminal GlcNAc residues in cell wall polymers (Lotan et al., 1975),
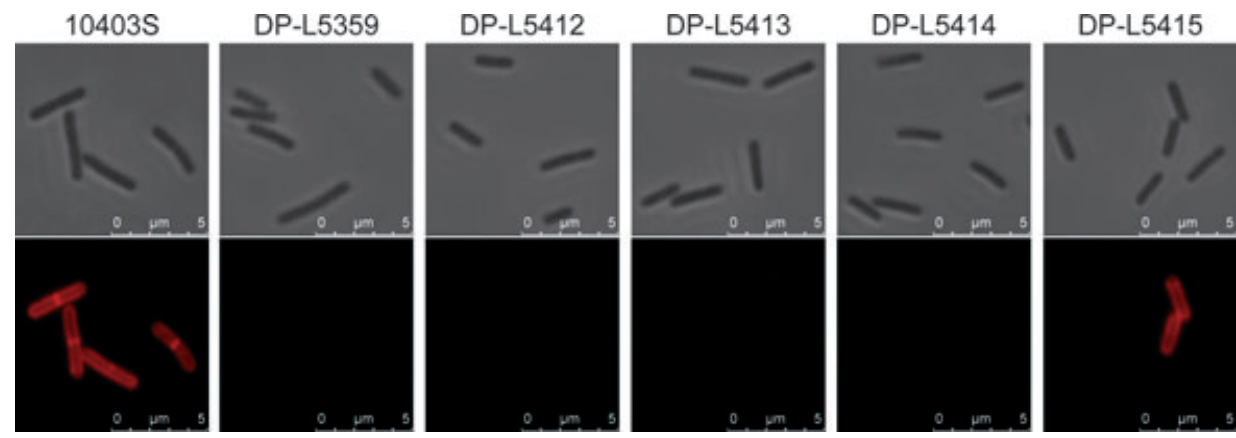

Fig. 4. Phase-contrast and corresponding fluorescence microscopy images for the detection of $N$-acetylglucosamine (GICNAC) on WTA of Listeria strains 10403S, DP-L5359, and complemented strains DP-L5412, DP-L5413, DP-L5414 and DP-L5415 using Alexa Fluor 594-labeled WGA. WGA recognizes GICNAC substitutes on WTA of Listeria monocytogenes. Partial restoration of 104035 is observed with complementation with LptpA2 (DP-L5415). 


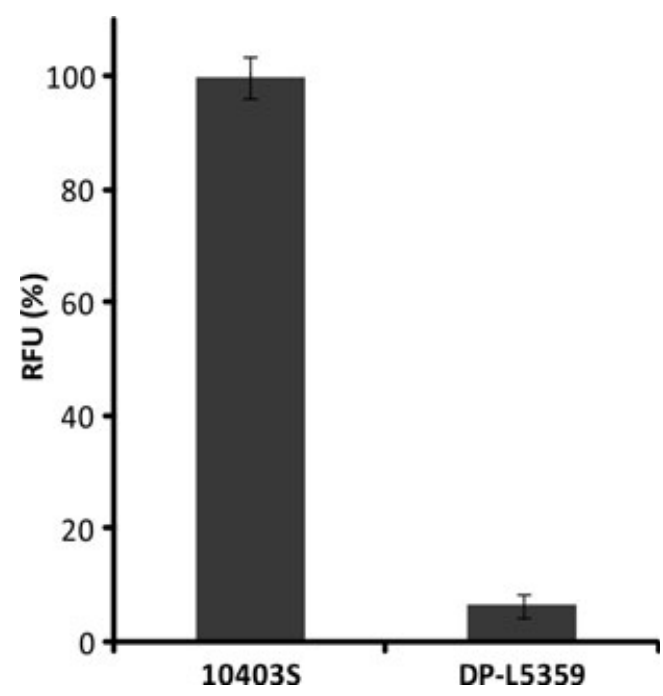

Fig. 5. Quantitative assessment of terminal GlcNAc residues in WTA by binding of GFP-tagged CBDP35 (HGFP-CBDP35). Purified cell walls of Listeria monocytogenes strains 10403 S and DP-L5359 were incubated with HGFP-CBDP35 and analyzed for binding by quantitative fluorescence analysis. Binding is measured in relative fluorescence units (RFU), and the amount of HGFP-CBDP35 bound to cell walls of strain 104035 is defined as $100 \%$. Measurements were taken in triplicate. Using this approach, the DP-L5359 was shown to have GIcNAc-deficient WTA.

was performed to test for the presence of GlcNAc in WTA. Alexa Fluor $594^{\circledR}$ WGA was able to stain WT strain 10403S and DP-L5415, but this lectin failed to bind to strains DP-L5359, DP-L5412, DP-L5413, and DPL5414, pointing to a lack of GlcNAc residues in WTA (Fig. 4), which is restored in the DP-L5415 complemented with LMRG1707. The same results were obtained when binding assays were performed with GlcNAc-specific fluorescent P35 phage endolysin cell wall-binding domain HGFP-CBDP35 (Fig. 5).

\section{Discussion}

In this work, we have found that PTPs have an effect upon the composition of the Listeria cell wall. This is similar to many other bacteria including some pathogens (Grangeasse et al., 2007; Lacour et al., 2008; Bechet et al., 2009). In Gram-negative bacteria tyrosine kinases and phosphorylation were suggested to be involved in the production of emulsan in the nonpathogen Acinetobacter lwoffi (Nakar \& Gutnick, 2003) and capsular polysaccharide production in E. coli and a few other bacteria (Obadia et al., 2007). In Gram-positive bacteria, a machinery that includes tyrosine kinase and phosphatase was sug- gested to be involved in the synthesis and export of extracellular polysaccharides, such as S. aureus (Soulat et al., 2002; Olivares-Illana et al., 2008) and S. pneumoniae (Morona et al., 2002). Similarly, protein tyrosine phosphorylation in L. monocytogenes is associated with changes in teichoic acid. However, no homologous machinery of the related Gram-positive S. pneumoniae or $S$. aureus can be found in L. monocytogenes.

The change in teichoic acids of our four PTPs deletion mutant was the lack of $\mathrm{N}$-acetyl glucosamine (GlcNAc) in the WTA. This was demonstrated by the changes in susceptibility to Listeria phages and could almost completely be restored by functional LptpA2 and partially restored by LptpB1/lipA. The fact that phage A511 and the Ply of phage P35 bind GlcNAc in the WTA (Wendlinger et al., 1996; Eugster et al., 2011) confirms our observation. Because phage A118 adsorption is dependent on rhamnose decoration of WTA (Wendlinger et al., 1996), we did not observe any changes between the A118 binding comparing the WT and the DP-L5359 strain. The lack of GlcNAc in cell WTA was further confirmed by the lack of labeling with florescent WGA or HGFP-CBDP35.

Protein tyrosine phosphatases in Listeria (e.g. the conventional PTPs LptpB1/LipA and LptpB2) were shown before to have dual function as tyrosine phosphatases and phosphoinositide phosphatases (Beresford et al., 2010; Kastner et al., 2011). No function was previously suggested for the low molecular weight LptpA1 and LptpA2. The PTP LptpB1/LipA was suggested to contribute to the virulence of two Listeria strains in a mouse infection model without obvious changes in macrophage or epithelial cells' growth curve assays (Kastner et al., 2011). We obtained similar results in the cellular growth curves of the four PTP deletion mutant but did not observe major virulence defect in the in vivo mouse infection models using the 10403S WT strain.

To conclude, the major phenotype that we have observed associated with PTPs deletion in L. monocytogenes was changes in GlcNAc glycosylation of WTA. However, the precise role of the tyrosine phosphatases in the modification of this extracellular polysaccharide remains unclear. The fact that there are similar PTPs in other pathogenic bacteria emphasizes the importance of understanding the role of bacterial PTPs and tyrosine phosphorylation.

\section{Acknowledgements}

This work was supported in part by grants from the National Institutes of Health to Daniel A. Portnoy AI27655 and AI063302 and by the Legacy Heritage grant 1640/08 of the Israeli Science Foundation to R.N.-P. 


\section{References}

Bach H, Papavinasasundaram KG, Wong D, Hmama Z \& Av-Gay Y (2008) Mycobacterium tuberculosis virulence is mediated by PtpA dephosphorylation of human vacuolar protein sorting 33B. Cell Host Microbe 3: 316-322.

Bach H, Wong D \& Av-Gay Y (2009) Mycobacterium tuberculosis PtkA is a novel protein tyrosine kinase whose substrate is PtpA. Biochem J 420: 155-160.

Bechet E, Guiral S, Torres S, Mijakovic I, Cozzone AJ \& Grangeasse C (2009) Tyrosine-kinases in bacteria: from a matter of controversy to the status of key regulatory enzymes. Amino Acids 37: 499-507.

Beresford NJ, Mulhearn D, Szczepankiewicz B et al. (2009) Inhibition of MptpB phosphatase from Mycobacterium tuberculosis impairs mycobacterial survival in macrophages. J Antimicrob Chemother 63: 928-936.

Beresford NJ, Saville C, Bennett HJ, Roberts IS \& Tabernero L (2010) A new family of phosphoinositide phosphatases in microorganisms: identification and biochemical analysis. BMC Genomics 11: 457.

Camilli A, Tilney LG \& Portnoy DA (1993) Dual roles of plcA in Listeria monocytogenes pathogenesis. Mol Microbiol 8: 143-157.

Chao J, Wong D, Zheng X, Poirier V, Bach H, Hmama Z \& Av-Gay Y (2010) Protein kinase and phosphatase signaling in Mycobacterium tuberculosis physiology and pathogenesis. Biochim Biophys Acta 1804: 620-627.

Cozzone AJ (2005) Role of protein phosphorylation on serine/ threonine and tyrosine in the virulence of bacterial pathogens. J Mol Microbiol Biotechnol 9: 198-213.

Eugster MR \& Loessner MJ (2011) Rapid analysis of Listeria monocytogenes cell wall teichoic acid carbohydrates by ESI-MS/MS. PLoS ONE 6: e21500.

Eugster MR, Haug MC, Huwiler SG \& Loessner MJ (2011) The cell wall binding domain of Listeria bacteriophage endolysin PlyP35 recognizes terminal GlcNAc residues in cell wall teichoic acid. Mol Microbiol 81: 1419-1432.

Fiedler F, Seger J, Schrettenbrunner A \& Seeliger HPR (1984) The biochemistry of murein and cell-wall teichoic-acids in the genus Listeria. Syst Appl Microbiol 5: 360-376.

Grangeasse C, Obadia B, Mijakovic I, Deutscher J, Cozzone AJ \& Doublet P (2003) Autophosphorylation of the Escherichia coli protein kinase Wzc regulates tyrosine phosphorylation of Ugd, a UDP-glucose dehydrogenase. J Biol Chem 278: 39323-39329.

Grangeasse C, Cozzone AJ, Deutscher J \& Mijakovic I (2007) Tyrosine phosphorylation: an emerging regulatory device of bacterial physiology. Trends Biochem Sci 32: 86-94.

Grundner C, Perrin D, Hooft van Huijsduijnen R et al. (2007) Structural basis for selective inhibition of Mycobacterium tuberculosis protein tyrosine phosphatase PtpB. Structure 15: 499-509.

Guevara RE, Mascola L \& Sorvillo F (2009) Risk factors for mortality among patients with nonperinatal listeriosis in Los Angeles County, 1992-2004. Clin Infect Dis 48: 1507-1515.
Hodgson DA (2000) Generalized transduction of serotype 1/2 and serotype $4 \mathrm{~b}$ strains of Listeria monocytogenes. Mol Microbiol 35: 312-323.

Kastner R, Dussurget O, Archambaud C, Kernbauer E, Soulat D, Cossart P \& Decker T (2011) LipA, a tyrosine and lipid phosphatase involved in the virulence of Listeria monocytogenes. Infect Immun 79: 2489-2498.

Koul A, Choidas A, Treder M, Tyagi AK, Drlica K, Singh Y \& Ullrich A (2000) Cloning and characterization of secretory tyrosine phosphatases of Mycobacterium tuberculosis. J Bacteriol 182: 5425-5432.

Lacour S, Bechet E, Cozzone AJ, Mijakovic I \& Grangeasse C (2008) Tyrosine phosphorylation of the UDP-glucose dehydrogenase of Escherichia coli is at the crossroads of colanic acid synthesis and polymyxin resistance. PLOS ONE 3: e3053.

Lauer P, Chow MY, Loessner MJ, Portnoy DA \& Calendar R (2002) Construction, characterization, and use of two Listeria monocytogenes site-specific phage integration vectors. J Bacteriol 184: 4177-4186.

Loessner MJ \& Scherer S (1995) Organization and transcriptional analysis of the Listeria phage A511 late gene region comprising the major capsid and tail sheath protein genes cps and tsh. J Bacteriol 177: 6601-6609.

Loessner MJ, Schneider A \& Scherer S (1996) Modified Listeria bacteriophage lysin genes (ply) allow efficient overexpression and one-step purification of biochemically active fusion proteins. Appl Environ Microbiol 62: 3057-3060.

Loessner MJ, Inman RB, Lauer P \& Calendar R (2000) Complete nucleotide sequence, molecular analysis and genome structure of bacteriophage A118 of Listeria monocytogenes: implications for phage evolution. Mol Microbiol 35: 324-340.

Loessner MJ, Kramer K, Ebel F \& Scherer S (2002) C-terminal domains of Listeria monocytogenes bacteriophage murein hydrolases determine specific recognition and high-affinity binding to bacterial cell wall carbohydrates. Mol Microbiol 44: 335-349.

Lotan R, Sharon N \& Mirelman D (1975) Interaction of wheat-germ agglutinin with bacterial cells and cell-wall polymers. Eur J Biochem 55: 257-262.

Macek B, Gnad F, Soufi B, Kumar C, Olsen JV, Mijakovic I \& Mann M (2008) Phosphoproteome analysis of E. coli reveals evolutionary conservation of bacterial Ser/Thr/Tyr phosphorylation. Mol Cell Proteomics 7: 299-307.

Morona JK, Morona R, Miller DC \& Paton JC (2002) Streptococcus pneumoniae capsule biosynthesis protein $\mathrm{CpsB}$ is a novel manganese-dependent phosphotyrosine-protein phosphatase. J Bacteriol 184: 577-583.

Murli S, Watson RO \& Galan JE (2001) Role of tyrosine kinases and the tyrosine phosphatase SptP in the interaction of Salmonella with host cells. Cell Microbiol 3: 795-810.

Musumeci L, Bongiorni C, Tautz L et al. (2005) Lowmolecular-weight protein tyrosine phosphatases of Bacillus subtilis. J Bacteriol 187: 4945-4956. 
Nakar D \& Gutnick DL (2003) Involvement of a protein tyrosine kinase in production of the polymeric bioemulsifier emulsan from the oil-degrading strain Acinetobacter lwoffii RAG-1. J Bacteriol 185: 1001-1009.

Obadia B, Lacour S, Doublet P, Baubichon-Cortay H, Cozzone AJ \& Grangeasse C (2007) Influence of tyrosine-kinase Wzc activity on colanic acid production in Escherichia coli $\mathrm{K} 12$ cells. J Mol Biol 367: 42-53.

Olivares-Illana V, Meyer P, Bechet E et al. (2008) Structural basis for the regulation mechanism of the tyrosine kinase CapB from Staphylococcus aureus. PLoS Biol 6: e143.

Peleg A, Shifrin Y, Ilan O et al. (2005) Identification of an Escherichia coli operon required for formation of the O-antigen capsule. J Bacteriol 187: 5259-5266.

Portnoy DA, Jacks PS \& Hinrichs DJ (1988) Role of hemolysin for the intracellular growth of Listeria monocytogenes. J Exp Med 167: 1459-1471.

Schmelcher M, Shabarova T, Eugster MR, Eichenseher F, Tchang VS, Banz M \& Loessner MJ (2010) Rapid multiplex detection and differentiation of Listeria cells by use of fluorescent phage endolysin cell wall binding domains. Appl Environ Microbiol 76: 5745-5756.

Siegman-Igra Y, Levin R, Weinberger M et al. (2002) Listeria monocytogenes infection in Israel and review of cases worldwide. Emerg Infect Dis 8: 305-310.

Soulat D, Vaganay E, Duclos B, Genestier AL, Etienne J \& Cozzone AJ (2002) Staphylococcus aureus contains two lowmolecular-mass phosphotyrosine protein phosphatases. J Bacteriol 184: 5194-5199.

Valyasevi R, Sandine WE \& Geller BL (1990) The bacteriophage kh receptor of Lactococcus lactis subsp. cremoris $\mathrm{KH}$ is the rhamnose of the extracellular wall polysaccharide. Appl Environ Microbiol 56: 1882-1889.

Wendlinger G, Loessner MJ \& Scherer S (1996) Bacteriophage receptors on Listeria monocytogenes cells are the $\mathrm{N}$-acetylglucosamine and rhamnose substituents of teichoic acids or the peptidoglycan itself. Microbiology $\mathbf{1 4 2}(\mathrm{Pt} 4)$ : 985-992.

Wright CS (1984) Structural comparison of the two distinct sugar binding sites in wheat germ agglutinin isolectin II. J Mol Biol 178: 91-104.

Yuan M, Deleuil F \& Fallman M (2005) Interaction between the Yersinia tyrosine phosphatase YopH and its macrophage substrate, Fyn-binding protein, Fyb. J Mol Microbiol Biotechnol 9: 214-223.

Zhou B, He Y, Zhang X et al. (2010) Targeting mycobacterium protein tyrosine phosphatase B for antituberculosis agents. P Natl Acad Sci USA 107: 4573-4578.

\section{Supporting Information}

Additional Supporting Information may be found in the online version of this article:

Appendix S1. Supplementary materials and methods.

Fig. S1. Sensitivity of Listeria monocytogenes strains to Lysozyme.

Fig. S2. Growth curve in activated macrophages.

Fig. S3. Virulence in mice.

Fig. S4. Analysis of total cell wall phosphate content of wild-type strain 10403S and deletion mutant DP-L5359.

Table S1. Primers used in this study.

Table S2. List of the sequenced Listeria spp. and strains available at the NCBI database in which BLAST $\mathrm{P}$ analysis was presented in Table 2.

Please note: Wiley-Blackwell is not responsible for the content or functionality of any supporting materials supplied by the authors. Any queries (other than missing material) should be directed to the corresponding author for the article. 